OPEN ACCESS

Edited by:

Changiz Geula,

Northwestern University,

United States

Reviewed by:

Shelley J. Allen,

University of Bristol, United Kingdom

Marcus O. Grimm,

Saarland University, Germany

*Correspondence:

Sim K. Singhrao

sksinghrao@uclan.ac.uk

Received: 10 July 2017 Accepted: 02 October 2017 Published: 24 October 2017

Citation:

Pritchard $A B$, Crean S, Olsen I and Singhrao SK (2017) Periodontitis,

Microbiomes and their Role in Alzheimer's Disease.

Front. Aging Neurosci. 9:336. doi: 10.3389/fnagi.2017.00336

\section{Periodontitis, Microbiomes and their Role in Alzheimer's Disease}

\author{
Anna B. Pritchard ${ }^{1}$, StJohn Crean ${ }^{1}$, Ingar Olsen ${ }^{2}$ and Sim K. Singhrao ${ }^{1 *}$ \\ ${ }^{1}$ Dementia \& Neurodegenerative Diseases Research Group, Faculty of Clinical and Biomedical Sciences, School of Dentistry, \\ University of Central Lancashire, Preston, United Kingdom, ${ }^{2}$ Department of Oral Biology, Faculty of Dentistry, University of \\ Oslo, Oslo, Norway
}

As far back as the eighteenth and early nineteenth centuries, microbial infections were responsible for vast numbers of deaths. The trend reversed with the introduction of antibiotics coinciding with longer life. Increased life expectancy however, accompanied the emergence of age related chronic inflammatory states including the sporadic form of Alzheimer's disease (AD). Taken together, the true challenge of retaining health into later years of life now appears to lie in delaying and/or preventing the progression of chronic inflammatory diseases, through identifying and influencing modifiable risk factors. Diverse pathogens, including periodontal bacteria have been associated with $A D$ brains. Amyloid-beta $(A \beta)$ hallmark protein of $A D$ may be a consequence of infection, called upon due to its antimicrobial properties. Up to this moment in time, a lack of understanding and knowledge of a microbiome associated with AD brain has ensured that the role pathogens may play in this neurodegenerative disease remains unresolved. The oral microbiome embraces a range of diverse bacterial phylotypes, which especially in vulnerable individuals, will excite and perpetuate a range of inflammatory conditions, to a wide range of extra-oral body tissues and organs specific to their developing pathophysiology, including the brain. This offers the tantalizing opportunity that by controlling the oral-specific microbiome; clinicians may treat or prevent a range of chronic inflammatory diseases orally. Evolution has equipped the human host to combat infection/disease by providing an immune system, but Porphyromonas gingivalis and selective spirochetes, have developed immune avoidance strategies threatening the host-microbe homeostasis. It is clear from longitudinal monitoring of patients that chronic periodontitis contributes to declining cognition. The aim here is to discuss the contribution from opportunistic pathogens of the periodontal microbiome, and highlight the challenges, the host faces, when dealing with unresolvable oral infections that may lead to clinical manifestations that are characteristic for AD.

Keywords: Alzheimer's disease, infections, functional amyloids, microbiomes, periodontitis

\section{INTRODUCTION}

Alzheimer's disease (AD) is the most common example of dementia causing around $60 \%-80 \%$ of all cases (Gaugler et al., 2016). AD is characterized by cognitive deficit and has a complex, multifactorial etiology. The limited treatment options make it a challenging condition in which neuropsychiatrists/neurologists can do little to help their patients. The relentlessly downward 
course of the disease has impact on both the patient and their carers. Furthermore, the rising aging population and the predicted increase in the prevalence of the disease has immediate and long-term socioeconomic implications (Prince et al., 2014). Classically there are two forms of this neurodegenerative condition. The familial/early-onset form displaying an earlier manifestation, albeit in fewer $(<5 \%)$ AD cases is overall, more severe with increased functional loss and $A \beta$ deposits (Bekris et al., 2010). Susceptibility genes and their co-expressing environmental factors appear to exert influence over the sporadic/late-onset form, which accounts for the majority $(95 \%)$ of $\mathrm{AD}$ cases. $\mathrm{AD}$ has a bi-phasic criterion of diagnosis, which involves correlation of the clinical presentation with neuropathological examination, at post-mortem (Braak and Braak, 1995). Despite the difference between familial and sporadic $\mathrm{AD}$, the underlying neuropathology remains common to both forms.

The two diagnostic neuropathological hallmarks are numerous extracellular deposits of amyloid-beta ( $\mathrm{A} \beta$ plaques) and neurofibrillary tangles (NFTs) in the frontal cortex and the hippocampal areas of the brain (Braak and Braak, 1995). In the brain, $A \beta$ can take different physiological states (soluble monomers, dimers, insoluble $A \beta_{40 / 42}$ ), which eventually result in an insoluble, stable $\beta$-helical sheet structure in the form of two morphologically different plaques. The senile plaques are composed of densely packed $A \beta_{1-42}$, and known for their cytotoxicity in causing demise of surrounding neurons (McGeer et al., 2017). The NFTs, located within cortical neurons, are composed of paired helical filaments (PHFs) and tau protein, the latter of which undergoes posttranslational modification in the form of hyperphosphorylation.

In addition to the classic diagnostic hallmark proteins, is the apparently asymptomatic developing neuropathology. Biomarker studies using positron emission tomography for levels of $\mathrm{A} \beta$, and magnetic resonance imaging for brain volume, indicate that $\mathrm{AD}$ onset begins years before the clinical picture emerges (McGeer et al., 2017). Cerebral inflammation in the form of activated glia (microglia and astrocytes; Norden and Godbout, 2013), and $A \beta_{1-40}$ plaques are two of the many asymptomatic features seen in $\mathrm{AD}$. This implies that age-related priming of glia due to bacterial entry from hosts' dysbiotic microbiomes elsewhere in the body (oral, gastrointestinal (GI) tract) provide slow inflammatory damage. In support, oral pathogens, especially Porphyromonas gingivalis, thrive under toxic inflammatory conditions and if present, may dampen the early pathogenic effects of glial cell activation (Singhrao et al., 2015). This makes the findings of a recent retrospective cohort study highlighting a 10-year exposure to chronic periodontitis may lead to manifesting AD (Chen et al., 2017), very plausible. Whether inflammation precedes $A \beta$ or vice versa, is not clear; but considering $A \beta$ as an innate immune protein with its antimicrobial properties (Soscia et al., 2010; Kumar et al., 2016) would place infections upstream of this hallmark protein. A suggested sequence of $\mathrm{AD}$ pathophysiology would likely follow infections, $A \beta$ deposition and glial cell activation. These three events may also provide an explanation for the age related glial cell priming by genetic switches turning on, during life, in an age dependent manner (Jayadev et al., 2013; Fan et al., 2017; Wu et al., 2017).

If atrophy of soft and bony tissues classically refers to decreased cell size/cell loss, such an observation can equally, imply shrinkage as a marker post inflammation. Brain atrophy is also a feature of multiple sclerosis (Pérez-Cerdá et al., 2016), which potentially presents with similar immune (innate and adaptive) tissue response as that seen in periodontal disease (Di Benedetto et al., 2013; Olsen et al., 2016). In periodontitis, the localized bacterial accumulation results in stimulations that elicit inflammation and activation of the innate immune system. Influx of systemic inflammatory cells follows a short time afterwards by an adaptive immune cell response leading to tissue loss (Di Benedetto et al., 2013; Olsen and Singhrao, 2015; Olsen et al., 2016). Recent evidence suggests that the adaptive immune system may have an important role in suppressing AD neuropathology (Marsh et al., 2016; Olsen et al., 2016). It may therefore be that, with aging and a waning adaptive immune system, $\mathrm{AD}$ neuropathology may be more likely to be evident. Additional to this, both periodontal pathogens ( $P$. gingivalis and Treponema denticola) show weak responses for attracting systemic inflammatory cells (neutrophils, T/B cells) into the brain (Olsen et al., 2016). However, the concept of inflammation and macroscopic atrophic appearance (enlarged sulci and ventricles) unique to $\mathrm{AD}$ brains may offer similar clues for a pivotal and primary role of inflammation (Figure 1) at the organ level.

The atrophic appearance of $\mathrm{AD}$ brains corroborates inflammation and is a compelling indication of numerous bacteria/bacterial endo/exo/toxins and fungi/viruses observed in association with $\mathrm{A} \beta$ plaques (Hill et al., 2014; Itzhaki, 2016; Lukiw, 2016; Pistollato et al., 2016; Alonso et al., 2017; Harris and Harris, 2017; Jiang et al., 2017; Maheshwari and Eslick, 2017; Zhao et al., 2017). The large microbial biodiversity identified from post-mortem $\mathrm{AD}$ brain specimens could be because of the differences in age, diet, lifestyle, geographical environment and disease status, a limitation also recognized by the human microbiome project ${ }^{1}$. This places a greater onus on microbial virulence factor(s)/pathogen associated molecular patterns (PAMPs) than live microbes exerting a pathological effect with the common end-point of this neurodegenerative disease. An example of this is the detection of lipopolysaccharide (LPS) in $\mathrm{AD}$ brains with the resulting opsonization of LPS-producing bacteria by glial cells (Poole et al., 2013), and their direct binding with $A \beta$ plaques (Zhan et al., 2016; Zhao et al., 2017). Undoubtedly, LPS from the outer membrane of Gram-negative bacteria is a powerful pro-inflammatory PAMP. This may carry with it proteolytic enzymes (gingipains, peptidyl deiminases and carbonic anhydrases) and appendages such as fimbriae and curli fibers (curli are functional amyloids housed on the outer membrane of several prokaryotes) and other amyloid-like proteins (Table 1). In vivo experimental models have suggested LPS from oral, Gram negative bacteria having a role in chronic local inflammation (DiCarlo et al., 2001); A $\beta$ release (Sheng et al., 2003; Wu et al., 2017); worsened cognition

\footnotetext{
${ }^{1}$ http://www.hmpdacc.org/
} 


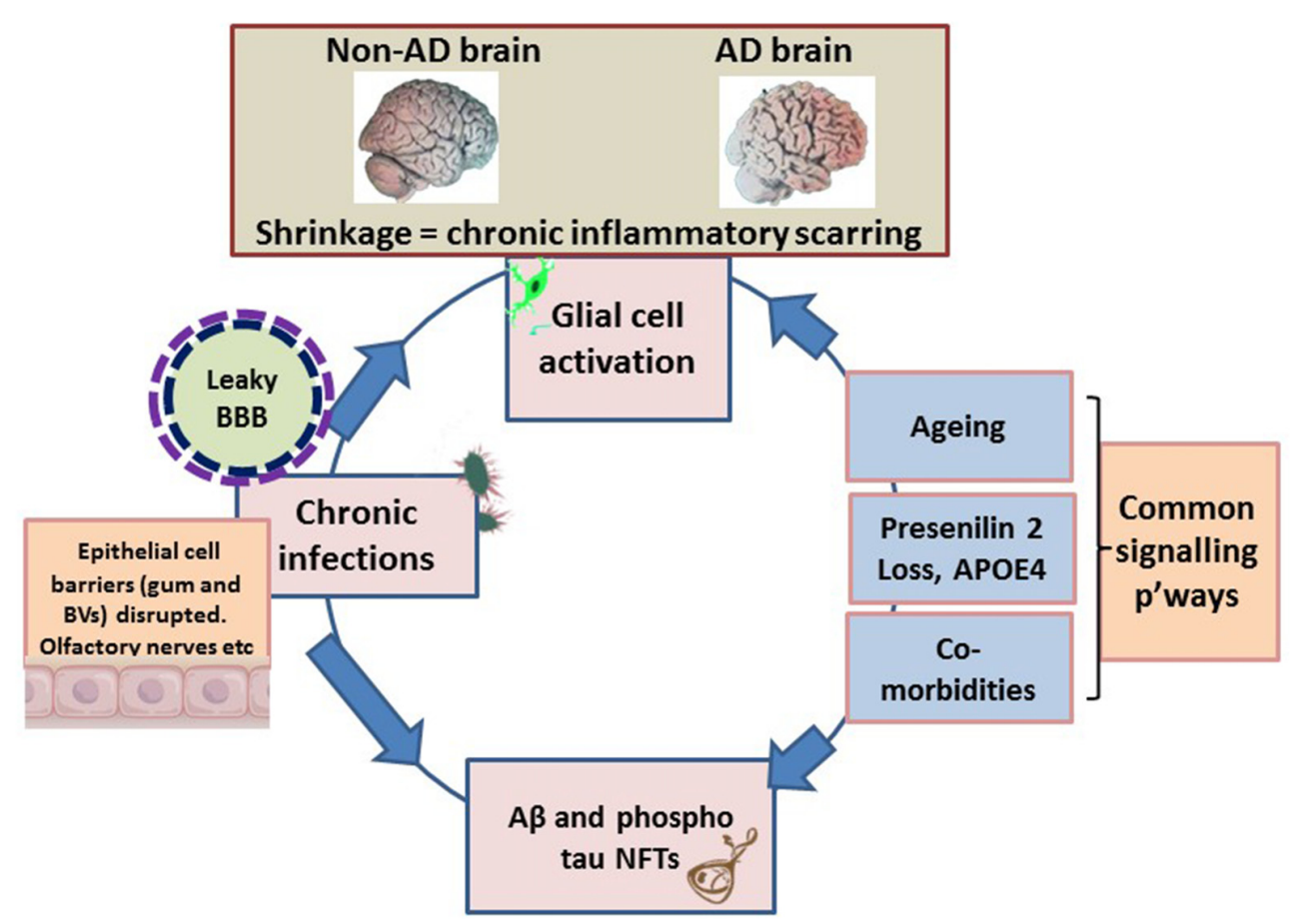

FIGURE 1 | Schematic illustrating the macroscopic features relating to shrinkage (wider sulci, compared with non-Alzheimer's disease (AD) brain), unique to the AD brain, which equates to inflammatory condition. The pathogens disrupt the epithelial cell-to-cell proteins of the gingivae through their proteases. The epithelial/endothelial barriers of capillaries disrupted for effective bacteremia to take place. The olfactory nerve pathways exploited to evade immune recognition. Environmental factors are the inflammophilic microbes with potential to subvert hosts immune defenses that also contribute to common inflammatory activities/pathways (p'ways) as well as contributing to proteostasis. At this stage the brain's resilience is markedly compromised and the blood-brain barrier (BBB) is becoming defective. The endotoxin intolerance/further inflammation tip the brain into disease.

TABLE 1 | Prokaryotes and eukaryotes found in biofilm communities with genes for expressing functional amyloids.

\begin{tabular}{llll}
\hline Species/Genera/Phylotypes & Protein & Protein function & References \\
\hline $\begin{array}{l}\text { Pseudomonas } \\
\text { Escherichia coli }\end{array}$ & FapC & Biofilm formation, virulence factor & Larsen et al. (2007) and Dueholm et al. (2010) \\
& CsgA, Curli & Biofilm formation, virulence factor & $\begin{array}{l}\text { Chapman et al. (2002), Dueholm et al. (2012) and } \\
\text { Zhan et al. (2016) }\end{array}$ \\
Klebsiella pneumoniae & Microcin E492 & Cytotoxicity & Bieler et al. (2005) \\
Mycobacterium tuberculosis & MTP & Adhesive pili & Alteri et al. (2007) \\
Bacillus subtilis & TasA & Biofilm & Romero et al. (2010, 2011, 2014) \\
Salmonella enterica & Curli & Biofilm, virulence factor & Solomon et al. (2005) \\
Salmonella typhimurium & Curli & Biofilm, virulence factor & Castelijn et al. (2012) \\
Streptococcus mutans & Adhesin P1 & Biofilm, caries & Oli et al. (2012) \\
Bacteroidetes & Curli & Biofilm & Larsen et al. (2008) and Dueholm et al. (2012) \\
Chloroflexi & Curli & Biofilm & Larsen et al. (2008) and Dueholm et al. (2012) \\
Firmicutes & Curli & Biofilm & Dueholm et al. (2012) \\
Thermodesulfobacteria & Curli & Biofilm & Dueholm et al. (2012) \\
Alpha, beta, delta and gamma-proteoabacteria & Curli & Biofilm & Larsen et al. (2007, 2008), Dueholm et al. (2012, \\
& & & 2013), \\
Candida albicans & Als & Adhesion & Ramsook et al. (2010) and Garcia et al. (2013) \\
\hline
\end{tabular}

(Wu et al., 2017); and tau protein phosphorylation (Lee et al., 2010). More pathogenic bacteria from the GI tract microbiome appear with curli fibers than presently known for the oral microbiome and therefore show direct associations with plaques (Lukiw, 2016; Pistollato et al., 2016; Zhan et al., 2016; Jiang et al., 2017; Zhao et al., 2017). A recent systematic review in which meta-analysis of periodontitis with $\mathrm{AD}$ was conducted, demonstrated a significant association between these two diseases (Odds Ratio (OR) 1.69, 95\% CI 1.21-2.35) and an even more significant association was observed in severe form of PD with AD (OR 2.98, 95\% CI 1.58-5.62; Leira et al., 2017). A population based retrospective study by Chen et al. (2017) demonstrated a 10 year, exposure of chronic periodontitis led to a higher risk (1.707-fold increase) of developing AD. 
Our aim here is to understand how the oral microbiome pathogens contribute to the hallmark proteins especially $\mathrm{A} \beta$ of $\mathrm{AD}$.

\section{THE ORAL MICROBIOME AND PERIODONTITIS}

Periodontal disease is one of the most common chronic polymicrobial infections in humans, characterized by loss of tooth supporting tissues due to the host's immuno-inflammatory responses, and consequently are a major cause of loss of teeth. Loss of more than 16 teeth in early to mid-life is significantly associated with the development of dementia with OR of 1.56 (95\% CI 1.12-2.18; Gatz et al., 2006; Luo et al., 2015). Conversely, retaining teeth and not brushing them, also exposes individuals to the risk of developing dementia. Taken together, the plausible explanation for missing and unclean teeth is poor oral hygiene (Paganini-Hill et al., 2012). Periodontitis is prevalent in individuals with poor oral hygiene and high dental plaque index.

The oral cavity is a home to over 700 different taxa and undoubtedly, this specific microbiome ${ }^{2}$ will keep growing, as changing bacterial species are included. At present, much smaller numbers $1 \times 10^{9} /$ pocket of mixed bacterial phyla (Loesche and Lopatin, 1998) are associated with periodontitis within this subgingival niche compared to the oral cavity where $1 \times 10^{11}$ bacteria/mg of dental plaque are recorded (Li et al., 2000). Dental plaque is a biofilm of a synergistic microbial community, and both genetic and environmental factors can cause it to become dysbiotic and lead to clinical manifestations of periodontitis. Some common examples of bacteria in the periodontal microbiome include $P$. gingivalis, Tannerella forsythia, Prevotella intermedia, Eikenella corrodens, Fusobacterium nucleatum, Aggregatibacter actinomycetemcomitans and T. denticola ${ }^{2}$. The Gram-negative bacterium $P$. gingivalis, is a keystone pathogen that modulates the dysbiosis of its companion species of bacteria beneath the gingivae (Hajishengallis and Lamont, 2014). The dysbiotic microbial community model of periodontal disease explains how a wide range and changing phyla can participate in generating pathology under the influence of this keystone pathogen (Hajishengallis and Lamont, 2016). The chronicity of periodontal disease, resulting in a potentially prolonged assault by a group of pathogens on the host's system, opens up the opportunity of specific microbes inducing a state where the host's threshold for disease exceeds that of health allowing local and remote organ pathology to develop.

\section{ORAL MICROBIOMES CONTRIBUTION TO AD}

Previous reports have alluded to the existence of an unexplored microbiome in the elderly and AD brains (Riviere et al., 2002), but the age at which infection takes hold in the brain is unknown. Microbiomes play an important role in balancing health and

${ }^{2}$ www.homd.org disease boundaries. Microbes (oral and non-oral) are implicated in the etiology of AD; this includes Borrelia species, T. denticola, P. gingivalis and Escherichia coli (Miklossy, 2011; Poole et al., 2013; Zhan et al., 2016), oral fungi (Carrasco et al., 2017) and others (Olsen and Singhrao, 2015; Maheshwari and Eslick, 2017). The diversity of microbes documented could be a reflection of the brain donor's geographical location, their age, diet, oral function (denture wearing) and lifestyle (Yatsunenko et al., 2012; Lukiw, 2013; Danborg et al., 2014; Heintz and Mair, 2014). Several scientists including us believe that the $\mathrm{AD}$ brain harbors its own microbiome (Emery et al., 2017), which may be due to the contribution of "radicalized" bacteria (Harding et al., 2017) from other human microbiomes (mouth, skin, GI tract), and as a consequence of the food chain and co-morbid states (Singhrao et al., 2016). Since age is the major risk factor for developing $\mathrm{AD}$, then evolving microbiomes may provide dynamics of the microbial communities over time. To this end, Brandscheid et al. (2017) examined changes in microbial communities in the GI tract of the 5XFAD AD transgenic mouse model and confirmed changes to microbial communities occurred over time. The changing microbes correlated with changes in trypsin secretions (Brandscheid et al., 2017), implying meat rich diets are indigestible in old age and excess protein may upset the existing microbial community dynamics. If two main phyla of GI tract bacteria are Firmicutes (approximately 80\%) and Bacteroidetes (approximately 20\%; Lukiw, 2016); it appears that during aging humans also undergo shifts in favor of Bacteroidetes in their GI tract microbiome (Pistollato et al., 2016). P. gingivalis is a species within the genus Bacteroides (within the phylum Bacteroidetes) and with periodontitis becoming more chronic and prevalent in old age, this would imply that the periodontal microbiome offers a relatively early indication of changing microbial dynamics.

An $\mathrm{AD}$-specific microbiome might be composed of bacteria from associated dysbiotic microbiomes because microbial infections explain the common inflammatory pathways (Olsen and Singhrao, 2015; Lukiw, 2016; Olsen et al., 2016) and their effects in the elderly brain via host's peripheral immune responses and related signaling pathways (see "LPS in AD Inflammatory Cascades" section). Undoubtedly, a complex etiology underlies the clinical manifestations seen in $\mathrm{AD}$ giving rise to new concepts. It is time to re-examine the infection model that asks: are microbes the causative agents of $\mathrm{AD}$ ? This pertinent question anxiously awaits answers (Fischer, 1910; MacDonald and Miranda, 1987; Miklossy, 1993; Mawanda and Wallace, 2013; Olsen and Singhrao, 2015; Itzhaki et al., 2016). To this end, the biofilm concept of AD senile plaques is proposed (Allen et al., 2016; Miklossy, 2016) and offers an alternative platform for answering this fundamental question.

\section{FUNCTIONAL AMYLOIDS FROM PROKARYOTIC ORIGINS}

The prokaryotic functional amyloid (Epstein and Chapman, 2008; Dueholm et al., 2013), affords diverse functions in biofilm communities that range from structural components namely, 
fimbriae, curli and other cellular appendages to act as oligomeric toxins (Larsen et al., 2007; Dueholm et al., 2013); reservoirs for quorum sensing; signaling molecules and binding of redox mediators (Dueholm and Nielsen, 2017). Furthermore, there is potential for these prokaryotic $A \beta$-like fibers to cross the bloodbrain barrier $(\mathrm{BBB})$ and form pathological senile plaques seen in AD. Presence of curli protein is emphasized by the GI tract bacteria (Pistollato et al., 2016; Jiang et al., 2017; Zhao et al., 2017) and as an analogy to prion plaques, help to explain the protein-protein interactions leading to senile plaque formation connecting the biofilm hypothesis (Allen et al., 2016; Miklossy, 2016).

Shahnawaz and Soto (2012), reported that the functional amyloid MccE492 from Klebsiella pneumoniae RYC492 has the ability to depolymerize from its fibrillary state and release oligomers capable of inducing cytotoxicity equivalent to pathological $\mathrm{A} \beta$ in $\mathrm{AD}$. This paved the way for the molecular mimicry theory (Hartman et al., 2013; Friedland, 2015). The molecular mimicry theory incorporates microbial curli fibers and human $A \beta$ as a protein-protein interaction, which can result in cross-seeding even if these proteins are dissimilar (Friedland, 2015). Additional prokaryotic and yeast functional amyloid systems and their sources are listed in Table 1 (Bian et al., 2000; Gophna et al., 2001; Larsen et al., 2007, 2008; Dueholm et al., 2010, 2012, 2013; Dueholm and Nielsen, 2017).

Infections induce acute phase proteins as part of the innate immune response (Gabay and Kushner, 1999). One of these is serum amyloid component P (SAP). SAP can bind Candida albicans (Klotz et al., 2016) in the similar way $\mathrm{A} \beta$ binds to bacteria in its antimicrobial peptide capacity (Soscia et al., 2010; Kumar et al., 2016). This implies that amyloid-like proteins (Table 1) from foreign and host sources undergo physical protein-protein interactions with potential to cross-seed with $\mathrm{A} \beta$, and increase the amyloid burden. These extrinsic proteins (curli) are PAMPs (Bian et al., 2000) with $\beta$-pleated sheet structures (Friedland, 2015), and they cross-react with antibodies to human A $\beta$ plaques (Miklossy, 2016). Due to their density and morphological appearance, the senile plaques may be composed of curli-like $A \beta_{1-42}$ compositions. Would it then be plausible to suggest that either $\mathrm{AD}$ is a result of mixed pathologies or it has multiple etiological agents (Figure 1) that bypass protective host barriers, whereby some give rise to $A \beta_{1-40}$ and others like curli fibers, cross-seed to generate $\mathrm{A} \beta_{1-42}$ ? The molecular mimicry/A $\beta$ cross-seeding hypothesis for bacterial phylotypes (Hartman et al., 2013; Friedland, 2015) is attractive as it gives microbes a more prominent role in $\mathrm{AD}$ causality.

\section{SENILE PLAQUE, A MINIATURE BIOFILM HYPOTHESIS}

The proposal that pathogenic microbes (including oral spirochetes) are able to manifest the pathological and biological hallmarks of AD led Allen et al. (2016) and Miklossy (2016) to propose that "the senile plaques" in syphilitic and Lyme disease brain specimens are conglomerates of pathogenic bacteria, and can be viewed as multispecies biofilms. This compelling notion supports the cross-reactivity of antibodies that detect the breakdown product of the amyloid precursor protein (APP) $\mathrm{A} \beta$ in human $\mathrm{AD}$ brains, with the "A $\beta$-like" senile plaques formed by spirochaetal aggregates in vitro (Miklossy, 2016). In support of the Allen and Miklossy biofilm hypothesis, Friedland (2015) proposes a mechanism in which curli fibers and/or other similar bacterial antigens, with capacity to aggregate and acquire $A \beta$ conformation would eventually grow by incorporating host $\mathrm{A} \beta$ and aggregate in the form of $\mathrm{AD}$ senile plaques (Friedland, 2015).

Hundreds of synergistic species of bacteria reside within the organ specific (mouth, skin, GI tract) biofilm ecologies that outnumber the entire cells making up the human body ${ }^{3}$. Given the diversity of microbes identified from AD brains, it is plausible to expect a heterogeneous biofilm in which bacteria, fungi and viruses, all reside side by side. Since it is established that $A \beta$ is an antimicrobial peptide (Soscia et al., 2010), an expected consequence to microbes would be that of death, specifically in the context of AD. This together with the already highly inflamed environment of the AD brain and ongoing glial cell activity is likely to kill bacteria and exclude brain abscesses forming. Therefore, the senile plaques of $\mathrm{AD}$ are only likely to retain bacterial virulence factors such as LPS and genomic DNA signatures. Some bacteria and fungi form spores within the $\mathrm{AD}$ brain tissue and that way, they bypass the antimicrobial effects of the $A \beta_{40 / 42}$. Microbiological culture based methodologies allow their detection as previously reported (Balin et al., 2008; Miklossy et al., 2017). This is a testimony to live microbes having entered the $\mathrm{AD}$ brain at some stage rather than mere contamination of tissue at postmortem. C. albicans has also been observed in post mortem AD brain specimens (Carrasco et al., 2017) and has functional amyloid-like adhesins (Als) on its cell surface (Ramsook et al., 2010; Garcia et al., 2013). C. albicans is an opportunistic yeast found commonly in the oral cavity, where it typically becomes pathogenic in immunosuppressed individuals or if local factors are conducive to its growth. For example, if the individual uses a steroid inhaler or wears dentures (Scully and Felix, 2005). C. albicans infection can be asymptomatic and is difficult to eradicate. The concept of trapping/incapacitating and killing bacteria in the brain by $A \beta$ antimicrobial activity (Soscia et al., 2010; Kumar et al., 2016) perhaps equally applies to SAP mediated trapping of C. albicans systemically (Klotz et al., 2016). However, this affinity could also be due to the amyloid-like adhesion on the surface of $C$. albicans (Ramsook et al., 2010; Garcia et al., 2013). If the senile plaques represent foci of miniature biofilms, then $C$. albicans may be responsible for adding to the $A \beta$ burden by bringing its own Als (Ramsook et al., 2010; Garcia et al., 2013) and the insoluble SAP from its primary niche (Ramsook et al., 2010; Klotz et al., 2016). As human brain $A \beta$ and bacterial LPS are resistant to degradation, their accumulation is to be expected.

\footnotetext{
${ }^{3}$ http://www.hmpdacc.org/
} 


\section{LPS in AD Proteostasis}

The predominant signaling cascades participating in the innate immune system in $\mathrm{AD}$ pathogenesis include the Toll-like receptor (TLR) pathways. LPS in rodents demonstrate participation of TLRs, CD14 and NF- $\kappa \mathrm{B}$ signaling cascades (Olsen and Singhrao, 2015; Lukiw, 2016) and indicates that acute phase inflammation is beneficial whilst chronic organ specific inflammation is detrimental (DiCarlo et al., 2001; Sheng et al., 2003; Lee et al., 2010; Herber-Jonat et al., 2011) E. coli is a member of the oral microbiome ${ }^{4}$. genetically encoded for curli protein, and is included here because of Friedland's protein-protein interaction hypothesis for its plausible contribution to AD senile plaques (Friedland, 2015). E. coli LPS (presumably with curli), also co-localizes with AD senile plaques (Zhan et al., 2016; Zhao et al., 2017). In addition, experiments with peripheral inoculations of LPS from $E$. coli in APPswe transgenic mice (Sheng et al., 2003) have demonstrated increased expression of APP with A $\beta$ release (Sheng et al., 2003). This result supports the concept of peripheral inflammation as an initiating factor in intracerebral inflammatory activity as well as supporting the release of at least one hallmark (A $\beta$ ) protein (Sheng et al., 2003). More recently, Wu et al. (2017) demonstrated that repeat injections of LPS from $P$. gingivalis, activated cathepsin $\mathrm{B}$ ( $\mathrm{a}$ form of $\beta$ secretase) indirectly to cleave APP intracellular fragmentation in an age-dependent manner. Cathepsin B plays a pivotal role in the neuroinflammation induced by $P$. gingivalis LPS followed by intracellular APP cleavage (Wu et al., 2017). Functional testing revealed that chronic and systemic administration of $P$. gingivalis LPS in middle-aged mice caused learning and memory deficits (Wu et al., 2017), supporting an $\mathrm{AD}$-like phenotype and giving this PAMP, from an oral keystone pathogen, a more prominent role in $\mathrm{AD}$ causality.

Salmonella species enter the oral cavity through consumption of contaminated meat and eggs (Edwards and Bruner, 1938). The importance of S. abortus equii (Edwards and Bruner, 1938) lies in its LPS in relation to neuroinflammation and more uniquely in tau protein phosphorylation. S. abortus equii LPS inoculations in the hippocampus of $\mathrm{rTg} 4510$ mice carrying the parental tau mutations and non-transgenic littermates (Lee et al., 2010), supported an initial neuroinflammatory activity, followed by increased Ser199/202 and phospho-tau Ser396 in the mutated group (Lee et al., 2010). This result demonstrates the role of several bacteria (host microbiomederived and extrinsic, food chain sources) and hosts' genetic susceptibility contributing to inflammatory stimuli subsequent to which tau phosphorylation (Lee et al., 2010) takes place. In other words, the signaling pathways participating in innate immune mediator release have the potential to modify the PHF bound tau protein by posttranslational means, in the vulnerable host, at any time. Thus, NFT formation could be both dependent and independent of $A \beta$ deposits.

${ }^{4}$ www.homd.org
With relevance to $\mathrm{AD}$ pathology, a defective $\mathrm{BBB}$ is documented (Montagne et al., 2015; Halliday et al., 2016) and a plausible explanation for the permeability is related to loss of cell-cell tight junctional proteins. LPS from some bacteria such as $P$. gingivalis also contains proteolytic enzymes (gingipains) that cleave and fragment proteins into smaller peptides. The proteolytic activity of gingipains also targets cell-cell adhesion molecules (Katz et al., 2000; Hintermann et al., 2002; Sheets et al., 2005). It is, therefore, likely that gingipains also contribute to the degradation of endothelial cell tight junction proteins, and contribute to loss of $\mathrm{BBB}$ functional integrity. In support of this hypothesis, $P$. gingivalis infected animal models demonstrated hippocampal damage via inflammation-mediated injury and IgG and gingipains in the cerebral microvasculature (Singhrao et al., 2017). In addition, the phagocytic oxidative burst of the host's neutrophils and macrophages at a much earlier time point of $P$. gingivalis infection, and the oxidative stress response initiated by bacteria can equally damage the hippocampal microvasculature (Rokad et al., 2017). A permeable BBB also has implications for entry of extra-cerebral amyloid/amyloidlike proteins to the brain and add to the existing amyloid burden. Furthermore, under appropriate conditions, arginine residues on the end of fragmented proteins can undergo citrullination, a form of posttranslational modification, initiated by $P$. gingivalis peptidyl arginine deiminase (Bielecka et al., 2014). This is particularly relevant for retaining C5a activity and attracting immune cells to the brain (Farkas et al., 2003; Bielecka et al., 2014). This may be why AD pathology lacks presence of systemic phagocytes and $T / B$ cells in $A D$ brains. The insoluble $A \beta_{40 / 42}$ however does associate with macromolecules such as DNA, LPS, metal ions and other binding proteins (Mueller-Steiner et al., 2006; Itzhaki, 2016; Maher et al., 2016; Zhan et al., 2016). An explanation for their binding to $A \beta$ could be to stabilize $\beta$-helical sheet structure formation.

\section{LPS in AD Inflammatory Cascades}

The prokaryotic functional amyloids and proteolytic enzymes have the ability to modulate and induce host responses, potentially playing a significant role in $\mathrm{AD}$ pathogenesis. For example, $P$. gingivalis gingipains also affect cellular functions related to immune signaling. The role of $P$. gingivalis negating the adaptive immune system relates to suppression of interleukin (IL-2) cytokine secretion (Olsen et al., 2016). The lack of IL-2 enhances innate and humoral immune responses resulting in a different cytokine profile. This changes the $\mathrm{T}$ helper 17 (Th17) cell lineage, in the modulation of the Th17/T-regulatory cell (Treg) clone formation. The result is an imbalance in Th17 and Treg populations as discussed elsewhere (Olsen et al., 2016). The predominant signaling cascades participating in the innate immune system in $\mathrm{AD}$ pathogenesis as mentioned earlier include CD14, TLRs and the NF- $\kappa$ B pathways (Lukiw, 2016), the cAMP-signaling pathway, the transformation growth factor-beta signaling pathway (TGF- $\beta$ ) and the p38 mitogen-activated protein kinase signaling (p38 MAPK) pathway. The latter signaling cascade 
mediates inflammatory and stress responses and is critical in regulating levels of multiple pro-inflammatory cytokines, such as tumor necrosis factor- $\alpha$ (TNF- $\alpha$ ), IL-1, IL-6 and IL-8, as well as enzymes involved in inflammatory cascades e.g., cyclooxygenase and inducible nitric oxide synthase (Chen et al., 1999; Underwood et al., 2000; Huang et al., 2004). TNF$\alpha$ cytokine is significantly upregulated in AD (Tarkowski et al., 1999), which could be the result of host's intrinsic genetic factors such as presenilin 2 and APOE4 allele inheritance (Jayadev et al., 2013; Fan et al., 2017), whilst co-morbidities from periodontitis and its etiological polymicrobial pathogens are also responsible for contributing to this cytokine pool (Kamer et al., 2009). A consequence of high levels of TNF- $\alpha$ is that, together with its converting enzyme, this cytokine can provide positive feedback between the $\gamma$-secretase site fragmentations of the APP into amyloid-alpha (A $\alpha$; Allen, 2017). Infections also induce oxidative stress and this too can have an impact on $A \alpha$ and $A \beta$ levels through the $\gamma$ - and $\beta$-secretase cleavage of APP (Tamagno et al., 2008). Some GI tract microbiome bacteria can alter $\gamma$-aminobutyric acid neurotransmitter signaling by metabolizing glutamate and this has direct implications for impaired cognition as described by Pistollato et al. (2016).

\section{FUTURE PERSPECTIVES}

From periodontal microbiome perspectives, and rightly so, much research has focused on making periodontitis an accepted modifiable risk factor, for $\mathrm{AD}$. The near future should recapitulate the $\mathrm{AD}$ hallmark protein formation with periodontal polymicrobial oral infections in $\mathrm{AD}$ transgenic models. The foreign amyloid-like proteins should be isolated and tested for their true potential to contribute to inflammation and protein-protein interactions leading to senile plaques in $\mathrm{AD}$. The results will open up tantalizing modifiable therapies whereby clinicians may treat or prevent a range of chronic inflammatory diseases orally through dental intervention, diet (probiotics) and education.

\section{CONCLUSION}

Undoubtedly, a complex etiology underlies the clinical manifestations seen in $\mathrm{AD}$. Candidate microbes conforming

\section{REFERENCES}

Allen, H. B. (2017). "Alzheimer's disease: assessing the role of spirochetes, biofilms, the immune system, and amyloid- $\beta$ with regard to potential treatment and prevention," in Handbook of Infection and Alzheimer's Disease, ed. J. Miklossy (Amsterdam: IOS Press), 83-88.

Allen, H. B., Morales, D., Jones, K., and Joshi, S. (2016). Alzheimer's disease: a novel hypothesis integrating spirochetes, biofilm, and the immune system. J. Neuroinfect. Dis. 7:200. doi: 10.4172/2314-7326.10 00200 to the $\mathrm{AD}$ microbiome would be those that induce immunosuppression, are pathogenic, are able to evade the innate and adaptive immune recognition, incite local inflammation and are incapable of allowing entry of activated peripheral blood myeloid cells in the brain. The periodontal microbiome does concur with the type of expected bacteria in $\mathrm{AD}$ brains. As an analogy to the dysbiotic periodontal microbial communities driving periodontal disease, the $\mathrm{AD}$ microbiome may reflect similar traits. One such example is the keystone periodontal pathogen $P$. gingivalis, which is a master immune evader and an immunosuppressor of the host through IL-2 suppression. Although $P$. gingivalis lacks the curli gene, it has alternative inflammatory mechanisms to indirectly activate $\beta$ secretases and contribute to host derived $\mathrm{A} \beta$ as well as correlate with loss of mental function. A recent systematic review and a 16-year follow-up retrospective cohort study significantly link 10-year exposure to chronic periodontitis as a risk factor for AD. These reports, together with effort from other researchers firmly places periodontitis as a risk factor for AD. Alzheimer's Research UK charity, suggests that one third of all $\mathrm{AD}$ cases are preventable by reducing modifiable risk factors. This is equivalent to at least 198,000 people in the UK unnecessarily suffering from an untreatable, mental illness. With periodontitis and AD showing significant associations, preventative measures must include dental care as an intervention for all members of the society from an early age.

\section{AUTHOR CONTRIBUTIONS}

ABP initiated the review and wrote most of it. SKS and IO reviewed and corrected the finer details as $\mathrm{PhD}$ supervisors of ABP. SC provided critical feedback and funding.

\section{FUNDING}

This work was supported by the European Commission (FP7HEALTH-306029 “TRIGGER") (IO) and the University of Central Lancashire, UK (SKS, ABP).

\section{ACKNOWLEDGMENTS}

IO thanks the European Commission, SKS and ABP the University of Central Lancashire, UK.
Alonso, R., Pisa, D., Aguado, B., and Carrasco, L. (2017). Identification of fungal species in brain tissue from Alzheimer's disease by next-generation sequencing. J. Alzheimers Dis. 58, 55-67. doi: 10.3233/JAD-170058

Alteri, C. J., Xicohténcatl-Cortes, J., Hess, S., Caballero-Olín, G., Girón, J. A., and Friedman, R. L. (2007). Mycobacterium tuberculosis produces pili during human infection. Proc. Natl. Acad. Sci. U S A 104, 5145-5150. doi: 10.1073/pnas.0602304104

Balin, B. J., Little, C. S., Hammond, C. J., Appelt, D. M., Whittum-Hudson, J. A., Gérard, H. C., et al. (2008). Chlamydophila pneumoniae and the etiology of late-onset Alzheimer's disease. J. Alzheimers Dis. 13, 371-380. doi: 10.3233/jad2008-13403 
Bekris, L. M., Yu, C.-E., Bird, T. D., and Tsuang, D. W. (2010). Genetics of Alzheimer disease. J. Geriatr. Psychiatry Neurol. 23, 213-227. doi: 10.1177/0891988710383571

Bian, Z., Brauner, A., Li, Y., and Normark, S. (2000). Expression of and cytokine activation by Escherichia coli curli fibers in human sepsis. J. Infect. Dis. 181, 602-612. doi: 10.1086/315233

Bielecka, E., Scavenius, C., Kantyka, T., Jusko, M., Mizgalska, D., Szmigielski, B., et al. (2014). Peptidyl arginine deiminase from Porphyromonas gingivalis abolishes anaphylatoxin C5a activity. J. Biol. Chem. 289, 32481-32487. doi: 10.1074/jbc.C114.617142

Bieler, S., Estrada, L., Lagos, R., Baeza, M., Castilla, J., and Soto, C. (2005). Amyloid formation modulates the biological activity of a bacterial protein. J. Biol. Chem. 280, 26880-26885. doi: 10.1074/jbc.M502031200

Braak, H., and Braak, E. (1995). Staging of Alzheimer's disease-related neurofibrillary changes. Neurobiol. Aging 16, 271-278. doi: 10.1016/01974580(95)00021-6

Brandscheid, C., Schuck, F., Reinhardt, S., Schäfer, K.-H., Pietrzik, C. U., Grimm, M., et al. (2017). Altered gut microbiome composition and tryptic activity of the 5xFAD Alzheimer's mouse model. J. Alzheimers Dis. 56, 775-788. doi: 10.3233/JAD-160926

Carrasco, L., Alonso, R., Pisa, D., and Rabano, A. (2017). “Alzheimer's disease and fungal infection," in Handbook of Infection and Alzheimer's Disease, ed. J. Miklossy (Amsterdam: IOS Press), 281-294.

Castelijn, G. A. A., van der Veen, S., Zwietering, M. H., Moezelaar, R., and Abee, T. (2012). Diversity in biofilm formation and production of curli fimbriae and cellulose of Salmonella Typhimurium strains of different origin in high and low nutrient medium. Biofouling 28, 51-63. doi: 10.1080/08927014.2011.648927

Chapman, M. R., Robinson, L. S., Pinkner, J. S., Roth, R., Heuser, J., Hammar, M., et al. (2002). Role of Escherichia coli curli operons in directing amyloid fiber formation. Science 295, 851-855. doi: 10.1126/science.1067484

Chen, B.-C., Chen, Y.-H., and Lin, W.-W. (1999). Involvement of p38 mitogenactivated protein kinase in lipopolysaccharide-induced iNOS and COX-2 expression in J774 macrophages. Immunology 97, 124-129. doi: 10.1046/j.13652567.1999.00747.x

Chen, C.-K., Wu, Y.-T., and Chang, Y.-C. (2017). Association between chronic periodontitis and the risk of Alzheimer's disease: a retrospective, populationbased, mactched-cohort study. Alzheimers Res. Ther. 9:56. doi: 10.1186/s13195017-0282-6

Danborg, P. B., Simonsen, A. H., Waldemar, G., and Heegaard, N. H. (2014). The potential of microRNAs as biofluid markers of neurodegenerative diseases-a systematic review. Biomarkers 19, 259-268. doi: 10.3109/1354750X.2014. 904001

Di Benedetto, A., Gigante, I., Colucci, S., and Grano, M. (2013). Periodontal disease: linking the primary inflammation to bone loss. Clin. Dev. Immunol. 2013:503754. doi: 10.1155/2013/503754

DiCarlo, G., Wilcock, D., Henderson, D., Gordon, M., and Morgan, D. (2001). Intrahippocampal LPS injections reduce A $\beta$ load in APP+PS1 transgenic mice. Neurobiol. Aging 22, 1007-1012. doi: 10.1016/s0197-4580(01)00292-5

Dueholm, M. S., Albertsen, M., Otzen, D., and Nielsen, P. H. (2012). Curli functional amyloid systems are phylogenetically widespread and display large diversity in operon and protein structure. PLoS One 7:e51274. doi: 10.1371/journal.pone.0051274

Dueholm, M. S., Halkjaer, P., Chapman, M., and Otzen, D. (2013). "Functional amyloids in bacteria," in Amyloid Fibrils and Prefibrillar Aggregates: Molecular and Biological Properties, ed. D. E. Otzen (Germany: Wiley), 411-438.

Dueholm, M. S., and Nielsen, P. H. (2017). "Amyloids-a neglected child of the slime," in The Perfect Slime, Microbial Extracellular Polymeric Substances (EPS), eds H.-C. Flemming, T. R. Neu and J. Wingender (London: IWA Publishing), 113-134.

Dueholm, M. S., Petersen, S. V., Sønderkær, M., Larsen, P., Christiansen, G., Hein, K. L., et al. (2010). Functional amyloid in Pseudomonas. Mol. Microbiol. 77, 1009-1020. doi: 10.1111/j.1365-2958.2010.07269.x

Edwards, P. R., and Bruner, D. W. (1938). Two new Salmonella types isolated from fowls. J. Hyg. 38, 716-720. doi: 10.1017/s0022172400011566

Emery, D. C., Shoemark, D. K., Batstone, T. E., Waterfall, C. M., Coghill, J. A., Cerajewska, T. L., et al. (2017). 16S rRNA next generation sequencing analysis shows bacteria in Alzheimer's post-mortem brain. Front. Aging Neurosci. 9:195. doi: 10.3389/fnagi.2017.00195
Epstein, E. A., and Chapman, M. R. (2008). Polymerizing the fibre between bacteria and host cells: the biogenesis of functional amyloid fibres. Cell. Microbiol. 10, 1413-1420. doi: 10.1111/j.1462-5822.2008.01148.x

Fan, Y. Y., Cai, Q. L., Gao, Z. Y., Lin, X., Huang, Q., Tang, W., et al. (2017). $A P O E \& 4$ allele elevates the expressions of inflammatory factors and promotes Alzheimer's disease progression: a comparative study based on Han and She populations in the Wenzhou area. Brain Res. Bull. 132, 39-43. doi: 10.1016/j. brainresbull.2017.04.017

Farkas, I., Takahashi, M., Fukuda, A., Yamamoto, N., Akatsu, H., Baranyi, L., et al. (2003). Complement C5a receptor-mediated signaling may be involved in neurodegeneration in Alzheimer's disease. J. Immunol. 170, 5764-5771. doi: 10.4049/jimmunol.170.11.5764

Fischer, O. (1910). Die presbyophrene Demenz, deren anatomische Grundlage und klinische Abgrenzung. Z. Gesamte Neurol. Psychiatr. 3, 371-471. doi: 10.1007/bf02893605

Friedland, R. P. (2015). Mechanisms of molecular mimicry involving the microbiota in neurodegeneration. J. Alzheimers Dis. 45, 349-362. doi: 10.3233/JAD-142841

Gabay, C., and Kushner, I. (1999). Acute-phase proteins and other systemic responses to inflammation. N. Engl. J. Med 340, 448-454. doi: 10.1056/nejm199904293401723

Garcia, M., Lipke, P., and Klotz, S. (2013). Pathogenic microbial amyloids: their function and the host response. OA Microbiol. 1:2.

Gatz, M., Mortimer, J. A., Fratiglioni, L., Johansson, B., Berg, S., Reynolds, C. A., et al. (2006). Potentially modifiable risk factors for dementia in identical twins. Alzheimers Dement. 2, 110-117. doi: 10.1016/j.jalz.2006.01.002

Gaugler, J., James, B., Johnson, T., Scholz, K., and Weuve, J. (2016). Alzheimer's association report; Alzheimer's disease facts and figures. Alzheimers Dement. 12, 459-509. doi: 10.1016/j.jalz.2016.03.001

Gophna, U., Barlev, M., Seijffers, R., Oelschlager, T. A., Hacker, J., and Ron, E. Z. (2001). Curli fibers mediate internalization of Escherichia coli by eukaryotic cells. Infect. Immun. 69, 2659-2665. doi: 10.1128/iai.69.4.2659-2665.2001

Hajishengallis, G., and Lamont, R. J. (2014). Breaking bad: manipulation of the host response by Porphyromonas gingivalis. Eur. J. Immunol. 44, 328-338. doi: 10.1002/eji.201344202

Hajishengallis, G., and Lamont, R. J. (2016). Dancing with the stars: how choreographed bacterial interactions dictate nososymbiocity and give rise to keystone pathogens, accessory pathogens and pathobionts. Trends Microbiol. 24, 477-489. doi: 10.1016/j.tim.2016.02.010

Halliday, M. R., Rege, S. V., Ma, Q., Zhao, Z., Miller, C. A., Winkler, E. A., et al. (2016). Accelerated pericyte degeneration and blood-brain barrier breakdown in apolipoprotein E4 carriers with Alzheimer's disease. J. Cereb. Blood Flow Metab. 36, 216-227. doi: 10.1038/jcbfm.2015.44

Harding, A., Robinson, S., Crean, S., and Singhrao, S. K. (2017). Can better management of periodontal disease delay the onset and progression of Alzheimer's disease? J. Alzheimers Dis. 58, 337-348. doi: 10.3233/JAD-170046

Harris, S. A., and Harris, E. A. (2017). "Herpes simplex virus type I and other pathogens are key causative factors in sporadic Alzheimer's disease," in Handbook of Infection and Alzheimer's Disease, ed. J. Miklossy (Amsterdam: IOS Press), 241-278.

Hartman, K., Brender, J. R., Monde, K., Ono, A., Evans, M. L., Popovych, N., et al. (2013). Bacterial curli protein promotes the conversion of $\mathrm{PAP}_{248-286}$ into the amyloid SEVI: cross-seeding of dissimilar amyloid sequences. PeerJ. 1:e5. doi: $10.7717 /$ peerj. 5

Heintz, C., and Mair, W. (2014). You are what you host: microbiome modulation of the aging process. Cell 156, 408-411. doi: 10.1016/j.cell.2014.01.025

Herber-Jonat, S., Mittal, R., Gsinn, S., Bohnenkamp, H., Guenzi, E., and Schulze, A. (2011). Comparison of lung accumulation of cationic liposomes in normal rats and LPS-treated rats. Inflamm. Res. 60, 245-253. doi: 10.1007/s00011-0100260-y

Hill, J. M., Clement, C., Pogue, A. I., Bhattacharjee, S., Zhao, Y., and Lukiw, W. J. (2014). Pathogenic microbes, the microbiome, and Alzheimer's disease (AD). Front. Aging Neurosci. 6:127. doi: 10.3389/fnagi.2014.00127

Hintermann, E., Haake, S. K., Christen, U., Sharabi, A., and Quaranta, V. (2002). Discrete proteolysis of focal contact and adherens junction components in Porphyromonas gingivalis-infected oral keratinocytes: a strategy for cell adhesion and migration disabling. Infect. Immun. 70, 5846-5856. doi: 10.1128/iai.70.10.5846-5856.2002 
Huang, H., Rose, J. L., and Hoyt, D. G. (2004). p38 mitogen-activated protein kinase mediates synergistic induction of inducible nitric-oxide synthase by lipopolysaccharide and interferon- $\gamma$ through signal transducer and activator of transcription 1 Ser727 phosphorylation in murine aortic endothelial cells. Mol. Pharmacol. 66, 302-311. doi: 10.1124/mol.66. 2.302

Itzhaki, R. F. (2016). "Herpes and Alzheimer's disease: subversion in the central nervous system and how it might be halted," in Handbook of Infection and Alzheimer's Disease, ed. J. Miklossy (Amsterdam: IOS Press), 67-78.

Itzhaki, R. F., Lathe, R., Balin, B. J., Ball, M. J., Bearer, E. L., Braak, H., et al. (2016). Microbes and Alzheimer's disease. J. Alzheimers Dis. 51, 979-984. doi: 10.3233/JAD-160152

Jayadev, S., Case, A., Alajajian, B., Eastman, A. J., Möller, T., and Garden, G. A. (2013). Presenilin 2 influences miR146 level and activity in microglia. J. Neurochem. 127, 592-599. doi: 10.1111/jnc. 12400

Jiang, C., Li, G., Huang, P., Liu, Z., and Zhao, B. (2017). The gut microbiota and Alzheimer's disease. J. Alzheimers Dis. 58, 1-15. doi: 10.3233/JAD-161141

Kamer, A. R., Craig, R. G., Pirraglia, E., Dasanayake, A. P., Norman, R. G., Boylan, R., et al. (2009). TNF- $\alpha$ and antibodies to periodontal bacteria discriminate between Alzheimer's disease patients and normal subjects. J. Neuroimmunol. 216, 92-97. doi: 10.1016/j.jneuroim.2009.08.013

Katz, J., Sambandam, V., Wu, J. H., Michalek, S. M., and Balkovetz, D. F. (2000). Characterization of Porphyromonas gingivalis-induced degradation of epithelial cell junctional complexes. Infect. Immun. 68, 1441-1449. doi: 10.1128/iai.68.3.1441-1449.2000

Klotz, S. A., Sobonya, R. E., Lipke, P. N., and Garcia-Sherman, M. C. (2016). Serum amyloid $\mathrm{P}$ component and systemic fungal infection: does it protect the host or is it a Trojan Horse? Open Forum Infect. Dis. 3:ofw166. doi: $10.1093 /$ ofid/ofw166

Kumar, D. K. V., Choi, S. H., Washicosky, K. J., Eimer, W. A., Tucker, S., Ghofrani, J., et al. (2016). Amyloid- $\beta$ peptide protects against microbial infection in mouse and worm models of Alzheimer's disease. Sci. Transl. Med. 8:340ra72. doi: 10.1126/scitranslmed.aaf1059

Larsen, P., Nielsen, J. L., Dueholm, M. S., Wetzel, R., Otzen, D., and Nielsen, P. H. (2007). Amyloid adhesins are abundant in natural biofilms. Environ. Microbiol. 9, 3077-3090. doi: 10.1111/j.1462-2920.2007.01418.x

Larsen, P., Nielsen, J. L., Otzen, D., and Nielsen, P. H. (2008). Amyloid-like adhesins produced by floc-forming and filamentous bacteria in activated sludge. Appl. Environ. Microbiol. 74, 1517-1526. doi: 10.1128/AEM.02274-07

Lee, D. C., Rizer, J., Selenica, M. B., Reid, P., Kraft, C., Johnson, A., et al. (2010). LPS- induced inflammation exacerbates phospho-tau pathology in rTg4510 mice. J. Neuroinflammation 7:56. doi: 10.1186/1742-2094-7-56

Leira, Y., Domínguez, C., Seoane, J., Seoane-Romero, J., Pías-Peleteiro, J. M., Takkouche, B., et al. (2017). Is periodontal disease associated with Alzheimer's disease? A systematic review with meta-analysis. Neuroepidemiology 48, 21-31. doi: $10.1159 / 000458411$

Li, X., Kolltveit, K. M., Tronstad, L., and Olsen, I. (2000). Systemic diseases caused by oral infection. Clin. Microbiol. Rev. 13, 5476-5558. doi: 10.1128/cmr.13.4. $547-558.2000$

Loesche, W. J., and Lopatin, D. E. (1998). Interactions between periodontal disease, medical diseases and immunity in the older individual. Periodontol. 2000 16, 80-105. doi: 10.1111/j.1600-0757.1998.tb00117.x

Lukiw, W. J. (2013). Variability in miRNA abundance, speciation and complexity amongst different human populations and potential relevance to AD. Front. Cell. Neurosci. 7:133. doi: 10.3389/fncel.2013.00133

Lukiw, W. J. (2016). Bacteroides fragilis lipopolysaccharide and inflammatory signaling in Alzheimer's disease. Front. Microbiol. 7:1544. doi: 10.3389/fmicb. 2016.01544

Luo, J., Wu, B., Zhao, Q., Guo, Q., Meng, H., Yu, L., et al. (2015). Association between tooth loss and cognitive function among 3063 Chinese older adults: a community-based study. PLoS One 10:e0120986. doi: 10.1371/journal.pone. 0120986

MacDonald, A. B., and Miranda, J. M. (1987). Concurrent neocortical borreliosis and Alzheimer's disease. Hum. Pathol. 18, 759-761. doi: 10.1016/s0046$8177(87) 80252-6$

Maher, B. A., Ahmed, I. A., Karloukovski, V., MacLaren, D. A., Foulds, P. G., Allsop, D., et al. (2016). Magnetite pollution nanoparticles in the human brain. Proc. Natl. Acad. Sci. U S A 113, 10797-10801. doi: 10.1073/pnas.1605941113
Maheshwari, P., and Eslick, G. D. (2017). "Bacterial infection increases the risk of Alzheimer's disease: an evidence-based assessment," in Handbook of Infection and Alzheimer's Disease, ed. J. Miklossy (Amsterdam: IOS Press), 151-160.

Marsh, S. E., Abud, E. M., Lakatos, A., Karimzadeh, A., Yeung, S. T., Davtyan, H., et al. (2016). The adaptive immune system restrains Alzheimer's disease pathogenesis by modulating microglial function. Proc. Natl. Acad. Sci. U S A 113, E1316-E1325. doi: 10.1073/pnas.1525466113

Mawanda, F., and Wallace, R. (2013). Can infections cause Alzheimer's disease? Epidemiol. Rev. 35, 161-180. doi: 10.1093/epirev/mxs007

McGeer, P. L., Rogers, J., and McGeer, E. G. (2017). "Inflammation, anti-inflammatory agents and Alzheimer's disease: the last 22 years," in Handbook of Infection and Alzheimer's Disease, ed. J. Miklossy (Amsterdam: IOS Press), 11-15.

Miklossy, J. (1993). Alzheimer's disease-spirochetosis? Neuroreport 4, 841-848. doi: 10.1097/00001756-199307000-00002

Miklossy, J. (2011). Alzheimer's disease-a neurospirochetosis. Analysis of the evidence following Koch's and Hill's criteria. J. Neuroinflammation 8:90. doi: 10.1186/1742-2094-8-90

Miklossy, J. (2016). Bacterial amyloid and DNA are important constituents of senile plaques: further evidence of the spirochetal and biofilm nature of senile plaques. J. Alzheimers Dis. 53, 1459-1473. doi: 10.3233/JAD160451

Miklossy, J., Khalili, K., Gern, L., Ericson, R. L., Darekar, P., Bolle, L., et al. (2017). "Borrelia burgdorferi persists in the brain in chronic lyme neuroborreliosis and may be associated with Alzheimer diasease," in Handbook of Infection and Alzheimer's Disease, ed. J. Miklossy (Amsterdam: IOS Press), 67-78.

Montagne, A., Barnes, S. R., Sweeney, M. D., Halliday, M. R., Sagare, A. P., Zhao, Z., et al. (2015). Blood-brain barrier breakdown in the aging human hippocampus. Neuron 85, 296-302. doi: 10.1016/j.neuron.2014.12.032

Mueller-Steiner, S., Zhou, Y., Arai, H., Roberson, E. D., Sun, B., Chen, J., et al. (2006). Antiamyloidogenic and neuroprotective functions of cathepsin B: implications for Alzheimer's disease. Neuron 51, 703-714. doi: 10.1016/j. neuron.2006.07.027

Norden, D. M., and Godbout, J. P. (2013). Review: microglia of the aged brain: primed to be activated and resistant to regulation. Neuropathol. Appl. Neurobiol. 39, 19-34. doi: 10.1111/j.1365-2990.2012.01306.x

Oli, M. W., Otoo, H. N., Crowley, P. J., Heim, K. P., Nascimento, M. M., Ramsook, C. B., et al. (2012). Functional amyloid formation by Streptococcus mutans. Microbiology 158, 2903-2916. doi: 10.1099/mic.0.060855-0

Olsen, I., and Singhrao, S. K. (2015). Can oral infection be a risk factor for Alzheimer's disease? J. Oral Microbiol. 7:29143. doi: 10.3402/jom.v7.29143

Olsen, I., Taubman, M. A., and Singhrao, S. K. (2016). Porphyromonas gingivalis suppresses adaptive immunity in periodontitis, atherosclerosis and Alzheimer's disease. J. Oral Microbiol. 8:33029. doi: 10.3402/jom.v8.33029

Paganini-Hill, A., White, S. C., and Atchison, K. A. (2012). Dentition, dental health habits, and dementia: the Leisure World Cohort study. J. Am. Geriatr. Soc. 60, 1556-1563. doi: 10.1111/j.1532-5415.2012.04064.x

Pérez-Cerdá, F., Sánchez-Gómez, M. V., and Matute, C. (2016). The link of inflammation and neurodegeneration in progressive multiple sclerosis. Mult. Scler. Demyelinat. Disord. 1:9. doi: 10.1186/s40893-016-0012-0

Pistollato, F., Sumalla Cano, S., Elio, I., Masias Vergara, M., Giampieri, F., and Battino, M. (2016). Role of gut microbiota and nutrients in amyloid formation and pathogenesis of Alzheimer disease. Nutr. Rev. 74, 624-634. doi: 10.1093/nutrit/nuw023

Poole, S., Singhrao, S. K., Kesavalu, L., Curtis, M. A., and Crean, S. (2013). Determining the presence of periodontopathic virulence factors in short-term post-mortem Alzheimer's disease brain tissue. J. Alzheimers Dis. 36, 665-677. doi: 10.3233/JAD-121918

Prince, M., Knapp, M., Guerchet, M., McCrane, P., Prina, M., ComasHerrera, A., et al. (2014). Dementia UK: Update. 2nd Edn. London: Alzheimer's Society.

Ramsook, C. B., Tan, C., Garcia, M. C., Fung, R., Soybelman, G., Henry, R., et al. (2010). Yeast cell adhesion molecules have functional amyloid-forming sequences. Eukaryot. Cell 9, 393-404. doi: 10.1128/EC.00068-09

Riviere, G. R., Riviere, K., and Smith, K. (2002). Molecular and immunological evidence of oral Treponema in the human brain and their association with Alzheimer's disease. Oral Microbiol. Immunol. 17, 113-118. doi: 10.1046/j. 0902-0055.2001.00100.x 
Rokad, F., Moseley, R., Hardy, S. R., Chukkapalli, S., Crean, S., Kesavalu, L., et al. (2017). Cerebral oxidative stress and microvasculature defects in TNF- $\alpha$ expressing transgenic and Porphyromonas gingivalis-infected $\mathrm{ApoE}^{-/-}$mice. J. Alzheimers Dis. 60, 359-369. doi: 10.3233/jad-170304

Romero, D., Aguilar, C., Losick, R., and Kolter, R. (2010). Amyloid fibers provide structural integrity to Bacillus subtilis biofilms. Proc. Natl. Acad. Sci. U S A 107, 2230-2234. doi: 10.1073/pnas.0910560107

Romero, D., Vlamakis, H., Losick, R., and Kolter, R. (2011). An accessory protein required for anchoring and assembly of amyloid fibres in B. subtilis biofilms. Mol. Microbiol. 80, 1155-1168. doi: 10.1111/j.1365-2958.2011.07653.x

Romero, D., Vlamakis, H., Losick, R., and Kolter, R. (2014). Functional analysis of the accessory protein TapA in Bacillus subtilis amyloid fiber assembly. J. Bacteriol. 196, 1505-1513. doi: 10.1128/JB.01363-13

Scully, C., and Felix, D. H. (2005). Oral medicine-update for the dental practitioner. Red and pigmented lesions. Br. Dent. J. 199, 639-645. doi: $10.1038 /$ sj.bdj.4813017

Shahnawaz, M., and Soto, C. (2012). Microcin amyloid fibrils A are reservoir of toxic oligomeric species. J. Biol. Chem. 287, 11665-11676. doi: 10.1074/jbc. M111.282533

Sheets, S. M., Potempa, J., Travis, J., Casiano, C. A., and Fletcher, H. M. (2005). Gingipains from Porphyromonas gingivalis W83 induce cell adhesion molecule cleavage and apoptosis in endothelial cells. Infect. Immun. 73, 1543-1552. doi: 10.1128/iai.73.3.1543-1552.2005

Sheng, J. G., Bora, S. H., Xu, G., Borchelt, D. R., Price, D. L., and Koliatsos, V. E. (2003). Lipopolysaccharide-induced-neuroinflammation increases intracellular accumulation of amyloid precursor protein and amyloid $\beta$ peptide in APPswe transgenic mice. Neurobiol. Dis. 14, 133-145. doi: 10.1016/s0969-9961(03)00069-x

Singhrao, S. K., Chukkapalli, S., Poole, S., Velsko, I., Crean, S., and Kesavalu, L. (2017). Chronic Porphyromonas gingivalis infection accelerates the occurrence of age-related granules in ApoE $\mathrm{E}^{-/-}$mice. J. Oral Microbiol. 9:1270602. doi: 10.1080/20002297.2016.1270602

Singhrao, S. K., Harding, A., Chukkapalli, S., Olsen, I., Kesavalu, L., and Crean, S. (2016). Apolipoprotein E related co-morbidities and Alzheimer's disease. J. Alzheimers Dis. 51, 935-948. doi: 10.3233/JAD150690

Singhrao, S. K., Harding, A., Poole, S., Kesavalu, L., and Crean, S. (2015). Porphyromonas gingivalis: periodontal infection and its putative links with Alzheimer's disease. Mediators Inflamm. 2015:137357. doi: 10.1155/2015/137357

Solomon, E. B., Niemira, B. A., Sapers, G. M., and Annous, B. A. (2005). Biofilm formation, cellulose production, and curli biosynthesis by Salmonella originating from produce, animal, and clinical sources. J. Food Prot. 68, 906-912. doi: 10.4315/0362-028x-68.5.906
Soscia, S. J., Kirby, J. E., Washicosky, K. J., Tucker, S. M., Ingelsson, M., Hyman, B., et al. (2010). The Alzheimer's disease-associated amyloid $\beta$-protein is an antimicrobial peptide. PLoS One 5:e9505. doi: 10.1371/journal.pone.0009505

Tamagno, E., Guglielmotto, M., Aragno, M., Borghi, R., Autelli, R., Giliberto, L., et al. (2008). Oxidative stress activates a positive feedback between the $\gamma$-and $\beta$-secretase cleavages of the $\beta$-amyloid precursor protein. J. Neurochem. 104, 683-695. doi: 10.1111/j.1471-4159.2007.05072.x

Tarkowski, E., Blennow, K., Wallin, A., and Tarkowski, A. (1999). Intracerebral production of tumor necrosis factor- $\alpha$, a local neuroprotective agent, in Alzheimer disease and vascular dementia. J. Clin. Immunol. 19, 223-230. doi: 10.1023/A:1020568013953

Underwood, D. C., Osborn, R. R., Bochnowicz, S., Webb, E. F., Rieman, D. J., Lee, J. C., et al. (2000). SB 239063, a p38 MAPK inhibitor, reduces neutrophilia, inflammatory cytokines, MMP-9, and fibrosis in lung. Am. J. Physiol. Lung Cell. Mol. Physiol. 279, L895-L902.

Wu, Z., Ni, J., Liu, Y., Teeling, J. L., Takayama, F., Collcutt, A., et al. (2017). Cathepsin B plays a critical role in inducing Alzheimer's disease-like phenotypes following chronic systemic exposure to lipopolysaccharide from Porphyromonas gingivalis in mice. Brain Behav. Immun. 65, 350-361. doi: 10.1016/j.bbi.2017.06.002

Yatsunenko, T., Rey, F. E., Manary, M. J., Trehan, I., Dominguez-Bello, M., Contreras, M., et al. (2012). Human gut microbiome viewed across age and geography. Nature 486, 222-227. doi: 10.1038/nature11053

Zhan, X., Stamova, B., Jin, L. W., DeCarli, C., Phinney, B., and Sharp, F. R. (2016). Gram-negative bacterial molecules associate with Alzheimer disease pathology. Neurology 87, 2324-2332. doi: 10.1212/WNL.0000000000 003391

Zhao, Y., Jaber, V., and Lukiw, W. J. (2017). Secretory products of the human GI tract microbiome and their potential impact on Alzheimer's disease (AD): detection of lipopolysaccharide (LPS) in AD hippocampus. Front. Cell. Infect. Microbiol. 7:318. doi: 10.3389/fcimb.2017.00318

Conflict of Interest Statement: The authors declare that the research was conducted in the absence of any commercial or financial relationships that could be construed as a potential conflict of interest.

Copyright (c) 2017 Pritchard, Crean, Olsen and Singhrao. This is an open-access article distributed under the terms of the Creative Commons Attribution License (CC BY). The use, distribution or reproduction in other forums is permitted, provided the original author(s) or licensor are credited and that the original publication in this journal is cited, in accordance with accepted academic practice. No use, distribution or reproduction is permitted which does not comply with these terms. 